\title{
Acute Myocardial Infarction Due to Spontaneous Coronary Artery Dissection and Plaque Rupture
}

\author{
Sana Riaz ${ }^{1}$, Mostafa Vasigh ${ }^{1}$, Emad Mogadam ${ }^{2,}{ }^{3}$, Devjani Ganesan ${ }^{1}$, Debanik Chaudhuri ${ }^{4}$ \\ 1. Internal Medicine, State University of New York (SUNY) Upstate Medical University, Syracuse, USA 2. Cardiology, \\ State University of New York (SUNY) Upstate Medical University, Syracuse, USA 3. Cardiology, University of Southern \\ California, Los Angeles, USA 4. Interventional Cardiology, State University of New York (SUNY) Upstate Medical \\ University, Syracuse, USA
}

Corresponding author: Sana Riaz,riazs@upstate.edu

\section{Abstract}

Myocardial infarction (MI) can be secondary to atherosclerotic coronary artery disease (ACAD) and nonatherosclerotic coronary artery disease (NACAD). The common cause of NACAD in young females is spontaneous coronary artery dissection (SCAD). We present a case of SCAD and plaque rupture leading to MI.

Categories: Cardiology, Emergency Medicine, Internal Medicine

Keywords: chest pain, acute st-elevation myocardial infarction, spontaneous coronary artery dissection, mortality

\section{Introduction}

Spontaneous coronary artery dissection (SCAD) is a rare cause of acute coronary syndrome (ACS), with an angiographic incidence of $0.1 \%-1.1 \%$ and accounts for up to $35 \%$ of myocardial infarctions (MI) in women $\leqslant$ age $50[1,2]$. We present a case of a 45 -year-old female with no prior cardiac history admitted with chest pain and noted to have the rare co-existence of SCAD and atherosclerotic coronary artery disease (ACAD). Our case also highlights the importance of early recognition of ACS and prompt intervention leading to improved outcomes as noted in our patient.

\section{Case Presentation}

A 45-year-old female, active smoker with no prior cardiac disease presented with acute onset chest pain. She was in acute distress. The chest pain was central with radiation to the left arm and was associated with shortness of breath. She had no family history of MI at age less than 50 years and no sudden cardiac death. Vitals signs on admission: blood pressure $90 / 80 \mathrm{mmHg}$, heart rate 100 beats/minute, and respiratory rate 20 breaths/minute. The patient was alert but lethargic. Heart sounds were normal, and no murmurs or added sounds were appreciated. Auscultation of the lung revealed bilateral air entry on initial evaluation. Laboratory work on admission showed normal renal function, normal complete blood count, except for

Received 04/30/2020 Review began 05/04/2020 Review ended 05/04/2020 Published 05/11/2020

(c) Copyright 2020

Riaz et al. This is an open access article distributed under the terms of the Creative Commons Attribution License CC-BY 4.0., which permits unrestricted use, distribution, and reproduction in any medium, provided the original author and source are credited. leukocytosis of $13.6 \times 10^{3} / \mu \mathrm{L}$. She had elevated troponin $\mathrm{T}$ of $1.74 \mathrm{ng} / \mathrm{mL}$ and brain natriuretic peptide (BNP) of $8158 \mathrm{pg} / \mathrm{mL}$. Urine toxicology was negative. The electrocardiogram showed ST elevations in the anterior leads (Figure 1).

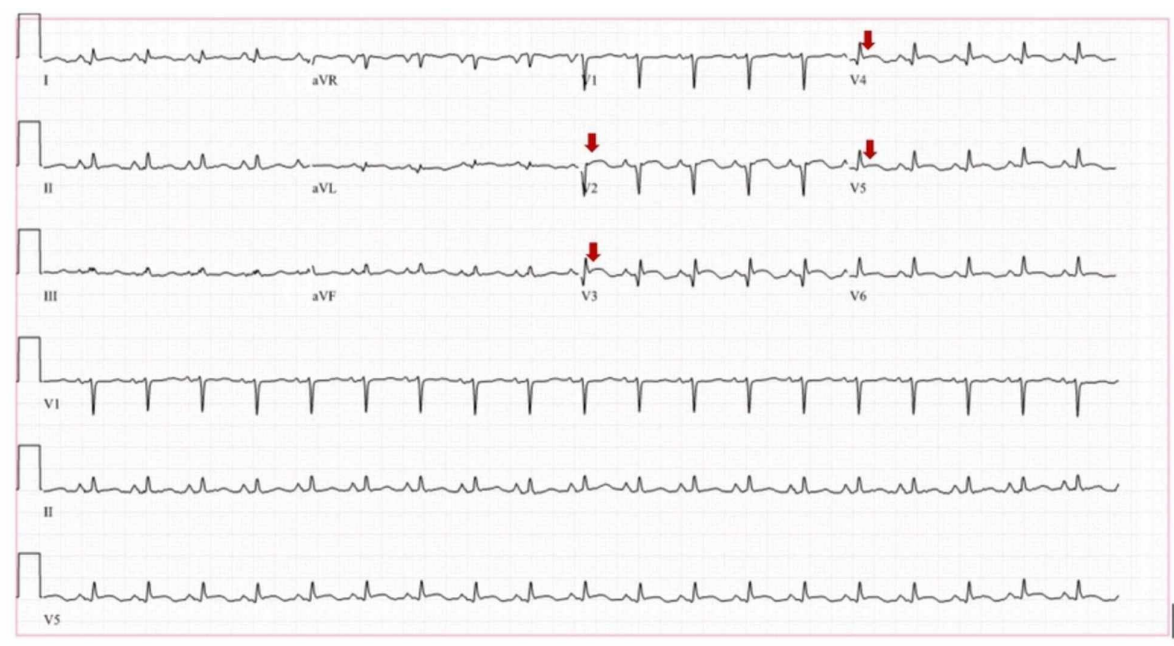

FIGURE 1: Electrocardiogram demonstrating ST-segment elevations in the anterior leads (arrows) 


\section{Cureus}

She was taken for emergent cardiac catheterization, which showed angiographically normal right coronary artery, a ruptured plaque with thrombus, and $90 \%$ stenosis involving the ostial left anterior descending artery (LAD) (Figure 2).

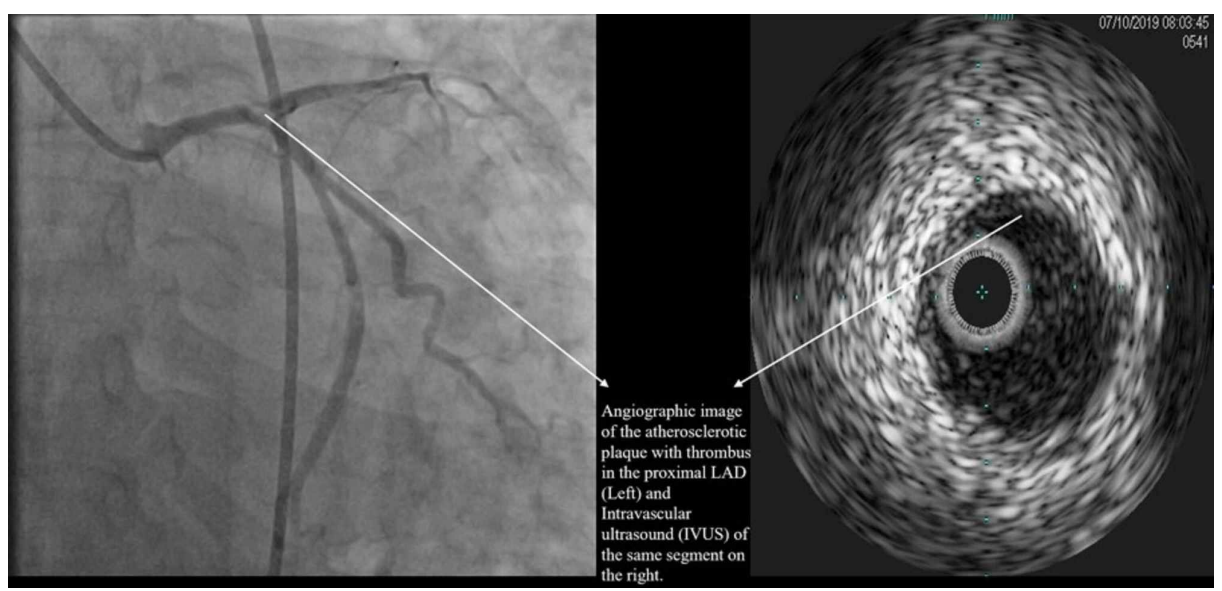

FIGURE 2: Proximal left anterior descending artery with atherosclerotic plaque and thrombus

LAD: Left anterior descending.

The mid-LAD showed diffuse narrowing, and apical LAD was completely occluded. The angiographic appearance of the lesions in mid-distal LAD was consistent with SCAD (Figure 3).

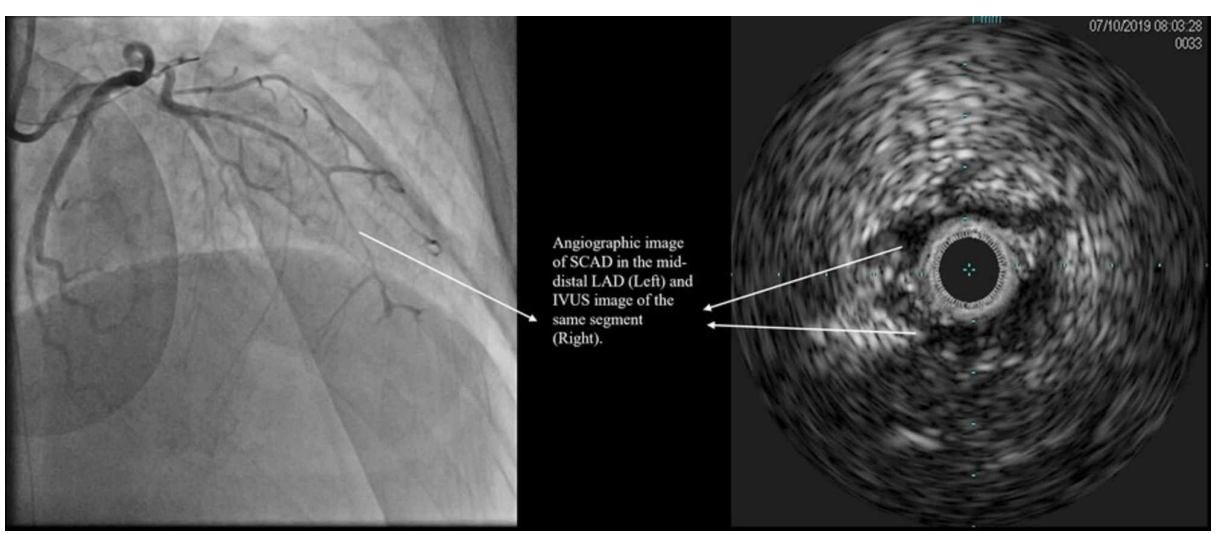

FIGURE 3: Spontaneous coronary artery dissection of the mid-distal left anterior descending artery

SCAD: Spontaneous coronary artery dissection; LAD: Left anterior descending; IVUS: Intravascular ultrasound.

This was subsequently confirmed on intravascular ultrasound (IVUS). The patient was in refractory cardiogenic shock (presence of low cardiac index and started on norepinephrine, vasopressin, and epinephrine) and required mechanical circulatory support (Impella, Aachen, Germany). A drug-eluting stent was placed in the proximal LAD. The apical occluded LAD was not crossed with a wire. The mid-LAD was treated with prolonged low-pressure balloon inflation.

Echocardiogram post- catheterization revealed akinetic anterior wall and an ejection fraction of $20 \%$ $25 \%$. She was gradually weaned off the vasopressors, and the Impella was removed 48 hours post-cardiac 
catheterization. She was medically optimized, and after hemodynamic stabilization, she was discharged home on dual antiplatelet therapy, high-intensity statin, lisinopril, and metoprolol succinate. Spironolactone was not initiated during the hospital stay due to low blood pressure.

The patient was followed up as an outpatient in two weeks and underwent a stress echocardiogram. Her left ventricular ejection fraction (LVEF) improved to 35\%-40\%, and she achieved 5.6 metabolic equivalents (METS). She was subsequently referred for cardiac rehabilitation and continues to follow with cardiology.

\section{Discussion}

Cardiovascular disease is the leading cause of death in women in the United States. The mortality due to MI in women younger than 50 years of age is two-fold higher than age-matched men. In previous studies, including a study by Saw et al., ACAD has been noted to be the most common etiology leading to MI [3]. However, non-atherosclerotic coronary artery disease (NACAD) is also an important cause for MI, and SCAD attributes to the majority of the NACAD cases [3]. SCAD is the dissection of the epicardial coronary artery that is not associated with atherosclerosis and trauma. Patients present with acute coronary syndrome (ACS) from coronary artery obstruction due to intramural hematoma rather than atherosclerotic plaque rupture or intraluminal thrombus. The LAD is the most affected artery ( $32 \%-46 \%$ of cases). Medical management of SCAD is similar to ACAD, however, the decision regarding percutaneous coronary intervention depends on multiple factors including perceived risk of complete coronary artery dissection and total occlusion and/or presence of hemodynamic compromise and cardiogenic shock [2,4]. It has been noted, based on a review by Saw et al., that the long term major adverse cardiac events (MACE) rates in patients with SCAD are $15 \%$ to $37 \%$ at five to seven years and almost $50 \%$ at 10 years [5]. Early recognition of SCAD is imminent since it is associated with a high mortality rate of about $50 \%$ [1].

Our case emphasizes the importance of a thorough evaluation of young women with no prior cardiac history and no cardiovascular risk factors presenting with acute onset chest pain. Since it has been observed that young women (less than 55 years of age) presenting with ACS, were most likely to be discharged from the emergency room with a missed diagnosis than other age groups or sex [3]. Our patient presented for the first time with chest pain, and on evaluation, she was found to have both SCAD and plaque rupture. However, appropriate and timely intervention can significantly decrease morbidity and mortality, as seen in our case [2]. The patient was stabilized and discharged home four days after her admission.

Additionally, our case is also unique since there are no case reports or literature reported with the coexistence of both SCAD and plaque rupture simultaneously leading to MI, based on our database search through PubMed and Google Scholar.

\section{Conclusions}

NACAD and ACAD are associated with risk factors; however, women under the age of 55 years without known risk factors for either entity should be evaluated when they present with acute onset chest pain. Additionally, risk stratification scores such as the history, ECG, age, risk factors, and troponin (HEART) score can facilitate decision making as it is also useful in patients with low risk of ACS.

\section{Additional Information \\ Disclosures}

Human subjects: Consent was obtained by all participants in this study. Conflicts of interest: In compliance with the ICMJE uniform disclosure form, all authors declare the following: Payment/services info: All authors have declared that no financial support was received from any organization for the submitted work. Financial relationships: All authors have declared that they have no financial relationships at present or within the previous three years with any organizations that might have an interest in the submitted work. Other relationships: All authors have declared that there are no other relationships or activities that could appear to have influenced the submitted work.

\section{References}

1. Pothiawala S, Lateef F: A case of spontaneous coronary artery dissection: it is not always plaque rupture . J Emerg Med. 2013, 44:92-95. 10.1016/j.jemermed.2011.08.024

2. Hayes SN, Kim ESH, Saw J, et al.: Spontaneous coronary artery dissection: current state of the science: a scientific statement from the American Heart Association. Circulation. 2018, 137:523-557. 10.1161/CIR.0000000000000564

3. Saw J, Aymong E, Mancini GBJ, Sedlak T, Starovoytov A, Ricci D: Nonatherosclerotic coronary artery disease in young women. Can J Cardiol. 2014, 30:814-819. 10.1016/j.cjca.2014.01.011

4. Rosengarten JA, Dana A: Recurrent spontaneous coronary artery dissection: acute management and literature review. Eur Hear J Acute Cardiovasc Care. 2012, 1:53-56. 10.1177/2048872612442404

5. Saw J, Mancini GBJ, Humphries KH: Contemporary review on spontaneous coronary artery dissection . J Am Coll Cardiol. 2016, 68:297-312. 10.1016/j.jacc.2016.05.034 